\title{
Local radiotherapy alone following neoadjuvant chemotherapy and surgery in combined clinical stage II and III breast cancer
}

\author{
Rohen White $2^{2^{*}}$, Tamara Dinneen ${ }^{2}$ and Andreas Makris ${ }^{1}$
}

\begin{abstract}
Purpose: The outcomes and recurrence patterns for patients with combined clinical stage II and III breast cancer treated with local but not regional radiotherapy after neoadjuvant chemotherapy (NAC) and surgery are poorly documented.

Methods: We performed a retrospective review of a prospectively collected database comprised of breast cancer patients who received NAC at our institution. 172 patients met the specified criteria of receiving NAC, surgery inclusive of axillary nodal dissection and post-operative local (but not regional) radiotherapy.

Results: One hundred eleven patients (64.5\%) were of combined clinical stage II and 61 (35.5\%) stage III at diagnosis. 103 patients (59.9\%) were clinically node positive with $101 \mathrm{cN1}$. On post-NAC pathology 29 (16.9\%) patients had a complete response, 30 (17.6\%) were combined yp stage I, 104 (60.5 \%) yp stage II and 9 (5.2\%) yp stage III. 77 (44.8\%) were node positive on post-NAC pathology, all ypN1. $52.3 \%$ were treated with breast conservation. At a median follow up of 67 months, 56 patients experienced breast cancer recurrence and 47 had died with breast cancer the dominant cause. Actuarial 5 and 10 year estimated freedom from locoregional recurrence (FFLRR), freedom from distant metastases (FFDM), disease free (DFS) and overall survival (OS) were 90 and 83.5, 74.5 and 64,69.5 and 56, 79.5 and 65 \% respectively. The most common pattern of failure was distant alone (without local or regional failure). Regional failure as the only site of first failure occurred in just three patients but was a component of first failure in a further twelve. Predictive factors on multivariate analysis for FFLRR were clinical stage II and estrogen receptor positivity. Prognostic factors were ypNO stage and estrogen receptor positive status.

Conclusions: Local radiotherapy alone may be reasonable for selected patients. Isolated distant recurrence is the dominant mode of failure for breast cancer patients who have received local radiotherapy without regional coverage following NAC.
\end{abstract}

Keywords: Breast cancer, Neoadjuvant chemotherapy, Regional radiotherapy, Patterns of recurrence

\section{Background}

Clinical indications for radiotherapy and target volumes following neoadjuvant chemotherapy (NAC) in the treatment of breast cancer are unclear [1-3]. Randomised controlled trial results from a non-NAC setting are often extrapolated to form the basis of radiotherapy recommendations but there is accumulating non-randomised evidence that this may result in over-treatment and

\footnotetext{
* Correspondence: rohenwhite@hotmail.com

${ }^{2}$ Breast Cancer Research Unit, Mt Vernon Cancer Centre, Middlesex, UK Full list of author information is available at the end of the article
}

unnecessary toxicity [4-6]. The uncertainty regarding the place of post-operative radiotherapy is highlighted in a patterns of management report from a recently published randomised controlled trial demonstrating much variation regardless of clinical or post NAC pathological stage [7].

At our centre practice is also not uniform. Postoperative radiotherapy following NAC and surgery for breast cancer is made on an individualized basis with many clinical oncologists adopting a local radiotherapy only approach to the conserved breast or chest wall. The rationale being that the risk of residual, microscopic 
regional disease post NAC in those with limited or no nodal disease on pathology is sufficiently low that it may be outweighed by the potential morbidity of regional radiotherapy.

The purpose of this study was to describe actuarial rates of recurrence from a breast cancer patient population treated with NAC, radical surgery and local radiotherapy to the conserved breast or chest wall. Recurrence patterns are detailed as well as predictive factors for freedom from locoregional recurrence (FFLRR) and overall survival (OS).

\section{Methods}

We conducted a retrospective analysis of a prospectively collected, single institution, NAC breast cancer database. Recruitment and data collection occurred between January of 1994 and December of 2013. All patients were retrospectively staged using the American Joint Committee on Cancer (AJCC) Staging Manual v7 [8]. For the purpose of the study the database was restricted to females of combined clinical stage II or III who received a breast conservation surgery or mastectomy inclusive of axillary node dissection, and received either chest wall or whole breast radiotherapy without dedicated regional radiotherapy. Patients were excluded if a breast cancer recurrence occurred prior to completion of adjuvant radiotherapy.

Within the database there is heterogeneity regarding systemic therapy regimens and much patient data predated routine human epidermal receptor 2 (HER2) amplification testing. In general, staging investigations, surgery and radiotherapy were consistent across the time period of patient recruitment.

Potential axillary involvement was clarified with ultrasound guided fine needle aspirate prior to NAC with minimal use of pre-NAC sentinel node biopsy. A level I and II axillary node clearance was standard after NAC.

Local breast or chest wall radiotherapy technique and dose prescriptions over the data collection period largely conformed to that subsequently described in the United Kingdom Standardisation of Breast Radiotherapy randomised controlled trial B which commenced accrual in 1999 [9]. Patients were simulated in the supine position (chest wall) or with a slight incline (whole breast) and the radiotherapy field edges marked $1.5-2 \mathrm{~cm}$ from the edge of breast tissue. In the setting of mastectomy, the contralateral breast was used to estimate breast landmarks and field edges. Earlier cases used 2D techniques without simulation computed tomography (CT) but overtime CT became mandatory. There was no use of contoured target volumes during the study period. Medial and lateral tangential, parallel opposed megavoltage beams of 6 or 10 megavoltage energy were used with dose prescribed to a point halfway between the lung and the skin surface on the perpendicular bisector of the posterior beam edge. Wedges were utilised to improve dose homogeneity. In CT plans unnecessary heart and lung were shielded with multi-leaf collimators and dose homogeneity optimised with the use of field-in-field techniques. Tangent arrangements were considered acceptable if there was less than $3 \mathrm{~cm}$ maximal lung and $1 \mathrm{~cm}$ maximal heart depth in-field.

Tumour bed boost prescription was clinician dependant and delineated at the time of simulation based on palpation of the surgical defect and position of the scar. Electrons were prescribed to D-max and a suitable energy chosen such that the $90 \%$ isodose was predicted to cover the tumour bed. The standard dose-fractionation schedules utilised in the United Kingdom over the database collection period were 40 Gy in 15 fractions and 50 Gy in 25 fractions to the chest wall or whole breast, treating once daily, five times per week. Tumour bed boost doses ranged from 10 Gy in 4 fractions to 16 Gy in 8 fractions and were also delivered once daily on consecutive weekdays. The use of chest wall bolus post mastectomy was clinician dependent and hence variable. Patients were restaged on suspicion of recurrence and all regions of recurrent disease documented as components of first recurrence.

Locoregional recurrence (LRR) was defined as the appearance of disease at one or more ipsilateral axillary, internal mammary or supraclavicular fossa nodal stations and/or ipsilateral chest wall or intact breast. This was further split into local (LR) and regional recurrence (RR). Distant metastasis (DM) was the appearance of breast cancer at any site outside of that considered LRR. Time-to-event endpoints were measured in months from diagnosis. Freedom from locoregional recurrence (FFLRR), freedom from distant metastasis (FFDM), disease free survival (DFS) and overall survival (OS) were estimated using reverse Kaplan Meier methods. Cox regression analysis was performed to assess for predictive and prognostic factors using a $p$ value below 0.05 as representing statistical significance. All statistical tests were performed on SPSS version 22.0.0.0 (IBM Corporation, Armonk, New York 2013).

\section{Results}

\section{Patient, tumour and treatment characteristics}

The database contained 713 patients of which 172 met the criteria for inclusion. The majority of exclusions were for clinical stage I disease or the utilisation of regional radiotherapy. Table 1 describes the patient, tumour and treatment characteristics. The median patient age at diagnosis was 49 years (range 27, 86).

Most patients received an anthracycline based NAC regimen without a taxane (56\%), nine percent received a taxane based regimen without an anthracycline and $31 \%$ percent received both. Two thirds of patients had HER-2 testing. Approximately $51 \%$ of patients were 
Table 1 Patient, tumour and treatment characteristics

\begin{tabular}{|c|c|c|c|}
\hline \multicolumn{2}{|l|}{ Characteristic } & \multirow{2}{*}{$\begin{array}{l}\begin{array}{l}\text { Number } \\
\text { (total = 172) }\end{array} \\
49(27,86)\end{array}$} & \multirow[t]{2}{*}{$\%$} \\
\hline Age & Median (range) & & \\
\hline \multirow[t]{2}{*}{ Age group } & $<50$ & 92 & $53.5 \%$ \\
\hline & $>=50$ & 80 & $46.5 \%$ \\
\hline \multirow[t]{2}{*}{ Laterality } & Right & 92 & $53.5 \%$ \\
\hline & Left & 80 & $46.5 \%$ \\
\hline \multirow[t]{3}{*}{ Breast location } & Outter quadrant & 95 & $55.2 \%$ \\
\hline & Inner and/or central & 59 & $34.3 \%$ \\
\hline & Unknown & 18 & $10.5 \%$ \\
\hline \multicolumn{4}{|l|}{ Clinical TNM stage ${ }^{a}$} \\
\hline \multirow[t]{3}{*}{ CT stage } & $0-2$ & 52 & $30.2 \%$ \\
\hline & 3 & 91 & $52.9 \%$ \\
\hline & 4 & 29 & $16.9 \%$ \\
\hline \multirow[t]{3}{*}{ cN stage } & 0 & 69 & $40.1 \%$ \\
\hline & 1 & 101 & $58.7 \%$ \\
\hline & 2 & 2 & $1.2 \%$ \\
\hline \multirow[t]{2}{*}{ combined c stage } & $\|$ & 111 & $64.5 \%$ \\
\hline & III & 61 & $35.5 \%$ \\
\hline \multirow[t]{2}{*}{ Inflammatory } & No & 153 & $89.0 \%$ \\
\hline & Yes & 19 & $11.0 \%$ \\
\hline \multirow[t]{3}{*}{ Histology } & Ductal carcinoma & 138 & $80.2 \%$ \\
\hline & Lobular carcinoma & 18 & $10.5 \%$ \\
\hline & Other & 16 & $9.3 \%$ \\
\hline \multirow[t]{3}{*}{ Grade } & 1 & 8 & $4.7 \%$ \\
\hline & 2 & 91 & $52.9 \%$ \\
\hline & 3 & 73 & $42.4 \%$ \\
\hline \multirow[t]{3}{*}{ ER status } & Negative & 64 & $37.2 \%$ \\
\hline & Positive & 101 & $58.7 \%$ \\
\hline & Unknown & 7 & $4.1 \%$ \\
\hline \multirow[t]{3}{*}{ HER2 status } & Negative & 81 & $47.1 \%$ \\
\hline & Amplified & 33 & $19.2 \%$ \\
\hline & Unknown & 58 & $33.7 \%$ \\
\hline \multirow[t]{4}{*}{ NAC regimen } & Anthracycline & 97 & $56.4 \%$ \\
\hline & Taxane & 16 & $9.3 \%$ \\
\hline & Both & 54 & $31.4 \%$ \\
\hline & Neither & 5 & $2.9 \%$ \\
\hline \multirow[t]{2}{*}{ Surgery type } & Lumpectomy & 90 & $52.3 \%$ \\
\hline & Mastectomy & 82 & $47.7 \%$ \\
\hline \multirow[t]{2}{*}{ Axillary nodes } & median (range) & $12(1,28)$ & \\
\hline & $<9$ nodes & 39 & $16.7 \%$ \\
\hline \multicolumn{4}{|c|}{ Pathological TNM stage ${ }^{a}$} \\
\hline \multirow[t]{3}{*}{ yp T stage } & $0-2$ & 154 & $89.5 \%$ \\
\hline & 3 & 13 & $7.6 \%$ \\
\hline & 4 & 2 & $1.2 \%$ \\
\hline
\end{tabular}

Table 1 Patient, tumour and treatment characteristics (Continued)

\begin{tabular}{llll}
\hline & unknown & 3 & $1.7 \%$ \\
yp N stage & 0 & 95 & $55.2 \%$ \\
combined yp stage & 1 & 77 & $44.8 \%$ \\
& pCR & 29 & $16.9 \%$ \\
& II & 30 & $17.4 \%$ \\
$>1$ node positive & III & 104 & $60.5 \%$ \\
& Yes & 9 & $5.2 \%$ \\
Trastuzumab use & No & 138 & $80.2 \%$ \\
& Yes & 34 & $19.8 \%$ \\
Radiotherapy schedule & $40 / 15$ & 146 & $84.9 \%$ \\
(Gray/\# fractions) & $50 / 25$ & 26 & $15.1 \%$ \\
& other & 158 & $91.9 \%$ \\
aTNM stage as per AJCC staging manual v7 & 11 & $6.4 \%$ \\
\hline
\end{tabular}

treated with breast conservation surgery inclusive of axillary dissection, $2 \%$ axillary dissection alone (for a putative primary) and the remainder modified radical mastectomy. The median number of nodes removed at axillary dissection was 12 (range 1,28).

On pre-NAC clinical staging all patients were either combined stage II or III. Only 2 patients (1.2\%) had clinical nodal stage greater than one. $16.9 \%$ of patients achieved a complete pathological response (pCR) having no microscopically identifiable invasive tumour in breast or axillary nodal tissue. The presence of ductal carcinoma in-situ only was considered a pCR. Whilst $35.5 \%$ were combined clinical stage III pre-NAC, only $5.7 \%$ were combined pathological stage III suggesting widespread downstaging. All patients were yp nodal stage $0(55 \%)$ or 1 (45\%).

The median follow-up for the group was 67 months (range 2, 248).

\section{Patterns of recurrence}

Fifty-six patients experienced recurrence at median time 30 months (range 47 to 153). The patterns of recurrence are presented in Fig. 1. LR, RR and DM were a component of first failure in 11 (6.4\%), 15 (8.7\%) and 48 (27.9\%) patients respectively. DM as the only site of failure was the most dominant pattern of failure, responsible for 36 events.

Isolated LRR occurred in eight patients of which isolated $R R$ occurred in three, isolated $L R$ in two and combined LR with RR in a further three. $R R$ as a component of first failure occurred in a further 12 patients. Multiple regional sites of first failure occurred in five patients and overall the supraclavicular fossa 


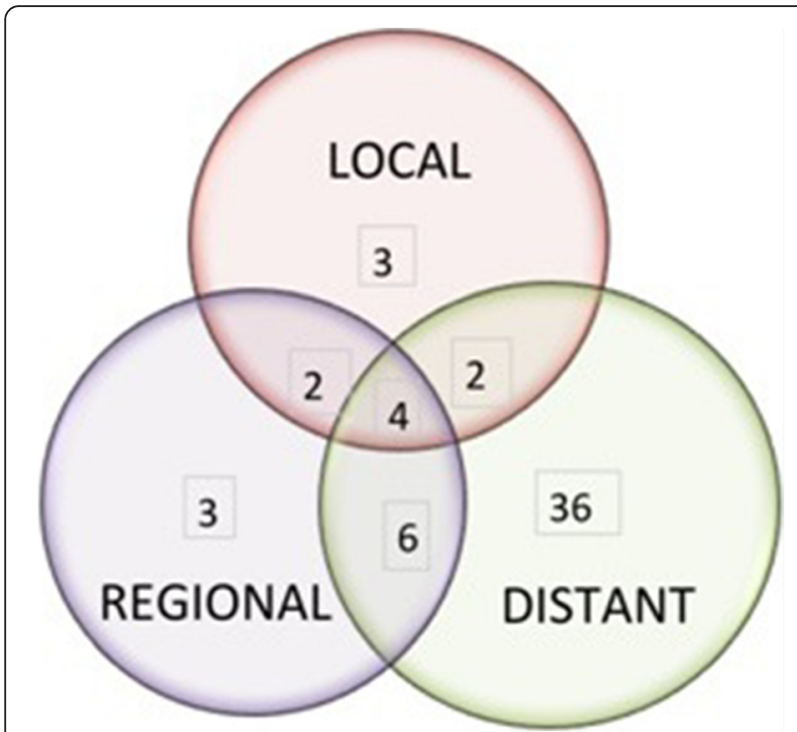

Fig. 1 Patterns of recurrence. 56 patients experienced recurrence. Local, regional and distant recurrence were a component of first failure in 11 (6.4\%), 15 (8.7\%) and 48 (27.9\%) patients respectively

(SCF), axilla and internal mammary nodal basins were components of first failure in 9, 6 and 5 patients respectively. Of the three isolated regional failures all involved the SCF with one additionally involving the axilla.

\section{Freedom from locoregional recurrence and distant metastases}

LRR as a component of first recurrence occurred in 20 patients and is illustrated in Fig. 2. The estimated actuarial rates of FFLRR at 5 and 10 years were $90(95 \%$ CI 85, 95) and $83.5 \%$ (95 \% CI 75.5, 91.5). The median time to LRR in those who experienced it was 27 months (range 9, 147).

DM as a component of first recurrence occurred in 48 patients. The estimated 5 and 10 year actuarial rates of FFDM were 74.5 (95 \% CI 67.5, 81.5) and $64 \%$ (95 \% CI $53,75)$ respectively.

\section{Disease free and overall survival}

Forty-seven patients died during the follow up period and the majority of deaths $(n=40)$ were related to breast cancer with 2 from other causes and 5 from causes unknown. A further 13 patients experienced recurrence but were alive at the time of last follow up. DFS is represented in Fig. 3 and OS in Fig. 4. The estimated 5 and 10 year DFS were 69.5 (95\% CI 62.5, 76.5) and $56 \%(95 \%$ CI 48, 64) respectively. The estimated OS at 5 and 10 years were $79.5(95 \%$ CI 73, 86) and $65 \%(95 \%$ CI 55, 75) respectively. Median time to death in those who experienced it was 44 months (range 7,177 ).

\section{Predictive factors for freedom from locoregional recurrence and overall survival}

Uni- and multivariate analysis are summarised in Table 2. On univariate analysis clinical stage II (versus clinical stage III) and estrogen receptor positivity predicted for FFLRR. On multivariate analysis they both remained statistically significant. On univariate analysis age less than 50 years, clinical stage II, cNO, ypNO and trastuzumab use were all statistically significant prognostic factors. On multivariable analysis estrogen receptor positivity and ypNO stage remained statistically significant.

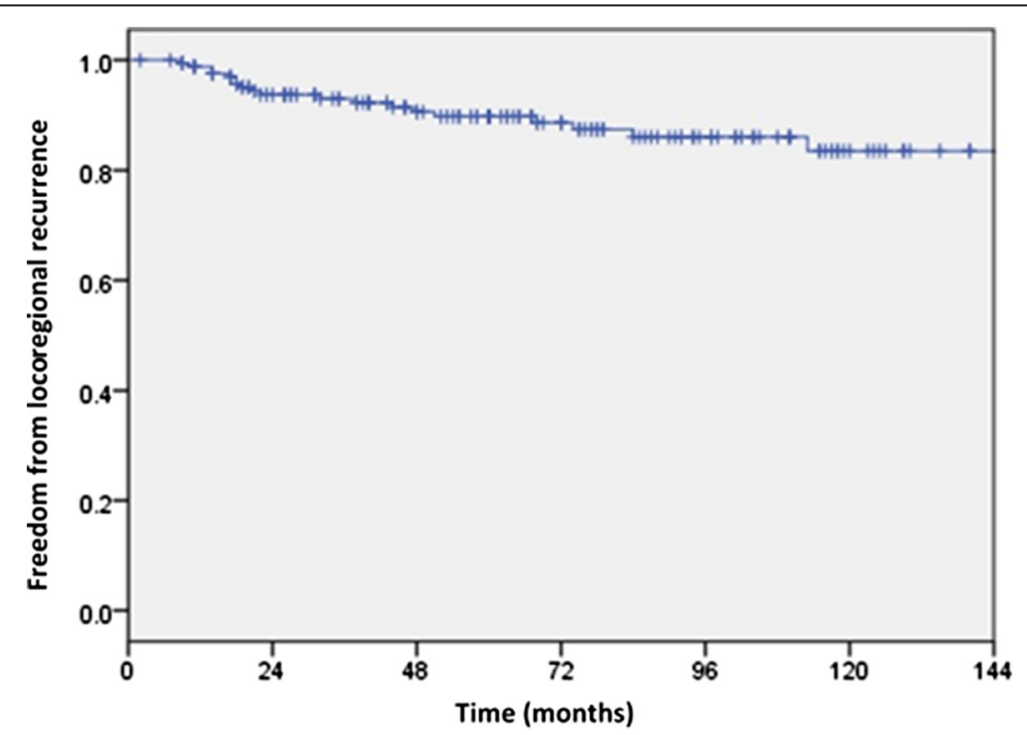

Fig. 2 Kaplan-Meier estimates of freedom from locoregional recurrence (FFLRR). Estimated actuarial rates of FFLRR at 5 and 10 years were 90 $(95 \% \mathrm{Cl} 85,95)$ and $83.5 \%(95 \% \mathrm{Cl} 75.5,91.5)$ 


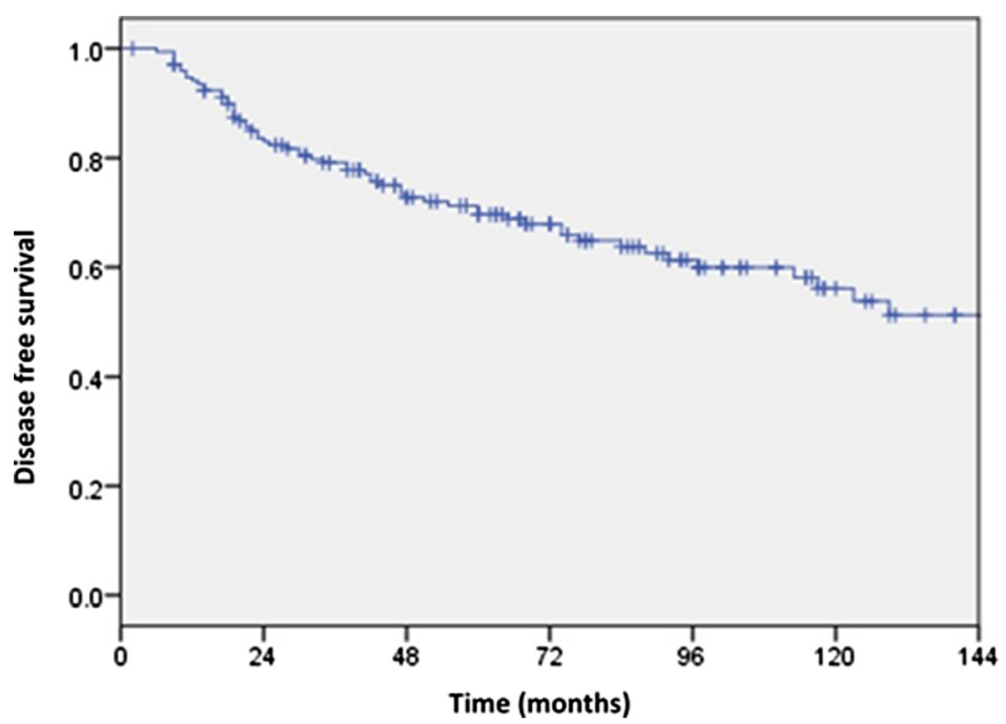

Fig. 3 Kaplan-Meier estimates of disease free survival (DFS). Estimated 5 and 10 year DFS were 69.5 (95\% Cl 62.5, 76.5) and $56 \%$ $(95 \% \mathrm{Cl} 48,64)$ respectively

\section{Discussion}

The role of post-operative radiotherapy following neoadjuvant chemotherapy for early breast cancer is uncertain and practice is far from uniform [7, 10]. A rationale for a local radiotherapy only approach can be made from recurrence data from landmark non-NAC post mastectomy randomised control trials reporting the vast majority of LRR as local, and from both prospective and retrospective studies reporting low locoregional recurrence rates in selected groups post NAC and mastectomy without adjuvant radiotherapy $[5,11-13]$. The recognised morbidity of post-operative dedicated regional radiotherapy in addition to local radiotherapy may hence outweigh its benefit in some. Patient data from our cohort suggests that selection for this radiotherapy program was dominated by patients who had a good response to NAC with almost all converting to combined yp stage 0 , I or II and only $20 \%$ having more than 1 lymph node involved.

Cross study comparison is difficult. The majority of modern series exploring the exclusion of post-operative radiotherapy after NAC are conducted exclusively in ypNO or PCR groups with arguably better outcomes $[3,5,6,14]$. Recurrence data from early NAC trials, prior to routine regional radiotherapy, report similar recurrence patterns in those who received breast conservation therapy and local radiotherapy despite consisting of only combined clinical stage I and II disease [11].

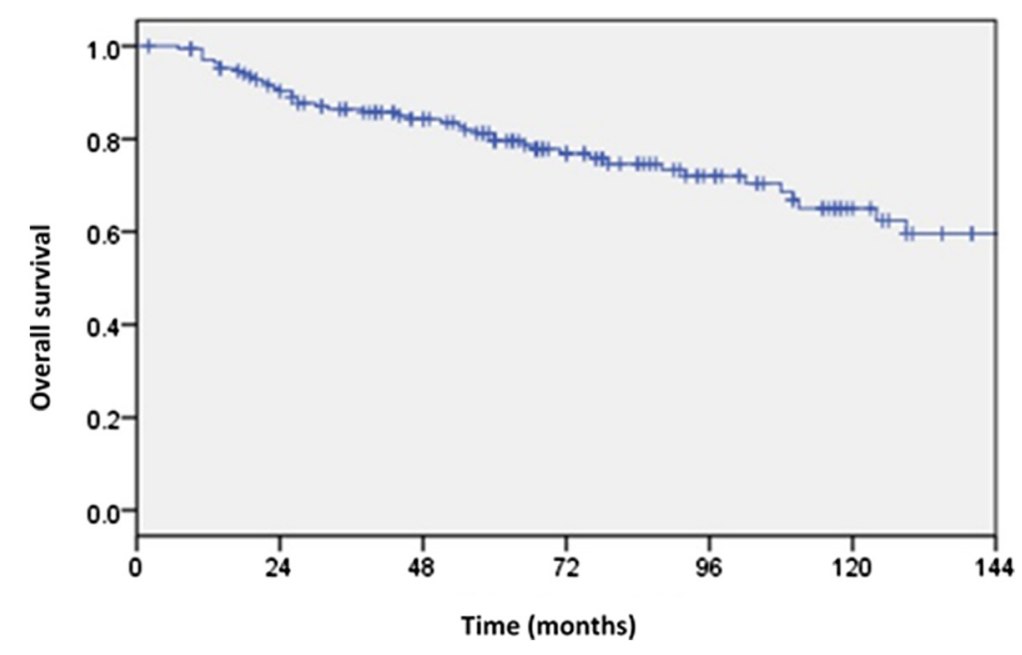

Fig. 4 Kaplan-Meier estimates of overall survival (OS). The estimated OS at 5 and 10 years were $79.5(95 \% \mathrm{Cl} 73,86)$ and $65 \%(95 \% \mathrm{Cl} 55,75)$ respectively 
Table 2 Univariate and multivariate analysis for freedom from locoregional recurrence (FFLRR) and overall survival (OS)

\begin{tabular}{|c|c|c|c|c|c|c|c|c|c|c|c|c|}
\hline \multirow[b]{3}{*}{ Factor } & & \multirow[b]{3}{*}{ Number } & \multicolumn{5}{|c|}{ Freedom from locoregional recurrence } & \multicolumn{5}{|c|}{ Overall Survival } \\
\hline & & & \multicolumn{2}{|l|}{ Univariate } & \multicolumn{3}{|c|}{ Multivariate } & \multicolumn{2}{|l|}{ Univariate } & \multicolumn{3}{|c|}{ Multivariate } \\
\hline & & & 5 yr FFLRR (\%) & $p$-value & $\mathrm{HR}$ & $(95 \% \mathrm{Cl})$ & $p$-value & 5 yr OS (\%) & $p$-value & $\mathrm{HR}$ & $(95 \% \mathrm{Cl})$ & $p$-value \\
\hline \multirow[t]{2}{*}{ Age } & $<50$ & 92 & 93.9 & 0.35 & & & & 83.7 & 0.02 & 1.90 & $(0.97-3.7)$ & 0.06 \\
\hline & $>=50$ & 80 & 84.9 & & & & & 75 & & & & \\
\hline \multirow[t]{2}{*}{ Combined c stage } & $\|$ & 111 & 94.5 & 0.00 & 5.51 & $(1.35-22.5)$ & 0.02 & 88.8 & 0.01 & 1.25 & $(0.58-2.7)$ & 0.56 \\
\hline & III & 61 & 81 & & & & & 62.7 & & & & \\
\hline \multirow[t]{2}{*}{ cN stage } & 0 & 69 & 93.6 & 0.21 & 1.00 & $(0.27-3.71)$ & 1.00 & 87.7 & 0.00 & 2.05 & $(0.92-4.55)$ & 0.08 \\
\hline & 1 & 101 & 87 & & & & & 74.2 & & & & \\
\hline \multirow[t]{2}{*}{ Inflammatory disease } & No & 153 & 90.2 & 0.09 & & & & 81.9 & 0.10 & 2.50 & $(0.96-6.52)$ & 0.06 \\
\hline & Yes & 19 & 84.7 & & & & & 58.5 & & & & \\
\hline \multirow[t]{2}{*}{ Grade } & 1 or 2 & 99 & 89.8 & 0.82 & & & & 80.8 & 0.39 & & & \\
\hline & 3 & 73 & 91.1 & & & & & 78.3 & & & & \\
\hline \multirow[t]{2}{*}{ Estrogen receptor } & Negative & 64 & 80 & 0.05 & 5.52 & $(1.84-16.55)$ & 0.00 & 72.2 & 0.14 & 2.50 & $(1.22-5.12)$ & 0.01 \\
\hline & Positive & 101 & 95.1 & & & & & 84.9 & & & & \\
\hline \multirow[t]{2}{*}{ Chemotherapy regimen } & Anthracyc & 97 & 90.7 & 0.22 & & & & 76.9 & 0.26 & & & \\
\hline & Anthracyc & 54 & 85.9 & & & & & 86.5 & & & & \\
\hline \multirow[t]{2}{*}{ Surgery } & $\mathrm{BCS}$ & 90 & 95.8 & 0.04 & 3.26 & $(0.97-10.94)$ & 0.06 & 81.9 & 0.76 & & & \\
\hline & MRM & 82 & 82.8 & & & & & 75.8 & & & & \\
\hline \multirow[t]{2}{*}{ Combined yp stage } & $0-\|$ & 163 & 89.9 & 0.97 & & & & 81 & 0.09 & & & \\
\hline & III & 9 & 87.5 & & & & & 55.6 & & & & \\
\hline \multirow[t]{2}{*}{$\mathrm{pCR}$} & No & 143 & 88.4 & 0.18 & & & & 77.2 & 0.13 & & & \\
\hline & Yes & 28 & 95.7 & & & & & 91.7 & & & & \\
\hline \multirow[t]{2}{*}{ ypN stage } & 0 & 95 & 89.3 & 0.94 & 1.30 & $(0.42-3.95)$ & 0.65 & 88.9 & 0.00 & 3.17 & $(1.33-7.15)$ & 0.01 \\
\hline & 1 & 77 & 90.1 & & & & & 69 & & & & \\
\hline \multirow[t]{2}{*}{$>1$ node involved } & No & 138 & 90.5 & 0.33 & & & & 82.3 & 0.06 & 1.26 & $(0.58-2.75)$ & 0.56 \\
\hline & Yes & 34 & 86.5 & & & & & 69.3 & & & & \\
\hline \multirow[t]{2}{*}{ Trastuzumab use } & No & 146 & 91.7 & 0.09 & & & 0.06 & 77.1 & 0.03 & 0.21 & $(0.03-1.53)$ & 0.12 \\
\hline & Yes & 26 & 79.3 & & & & & 93.3 & & & & \\
\hline
\end{tabular}

The subdata analysis unsurprisingly suggested that patients of earlier clinical stage, negative nodes and estrogen receptor positive phenotype faired best. The overall number of patients with a pathologically complete response and pathological stage III disease were likely too small for meaningful predictive analysis. The suggestion that breast conservation was favourable relative to mastectomy for FFLRR almost certainly reflects more advanced clinical disease receiving more intense therapy inclusive of mastectomy.

Isolated local recurrence $(n=2)$, isolated regional recurrence $(n=3)$ and locoregional without distant recurrence $(n=3)$ were uncommon. However, as a component of first failure regional recurrence was perhaps more frequent than expected $(n=15)$ with the supraclavicular fossa involved in nine of the fifteen regional recurrences (including all three isolated regional recurrences). This site would typically be covered by routine regional radiotherapy. It is not possible to discern the temporal relationship of combined site failures from this study but it is certainly tempting to hypothesize that distant recurrence in some patients may represent sequential seeding from an unsterilized regional site. In which case, the addition of regional radiotherapy may have impacted on disease outcomes. The National Surgical Adjuvant Breast and Bowel Project (NSABP) B-51 randomised controlled trial will assist in addressing this question for patients who convert to ypNO status post NAC.

The premise of omitting regional radiotherapy in this group of patients was that a potentially modest improvement in disease specific outcomes may be outweighed by treatment related toxicity. Whilst there is a theoretical increased risk of brachial plexopathy, thyroid dysfunction and potential second malignancy with regional radiotherapy in addition to chest or conserved breast the 
absolute increased risk is likely to be low and there is minimal literature to aid. Upper limb lymphedema is a commonly cited concern of regional radiotherapy but data from two recently published, high quality randomised control trials using 3D conformal techniques reported lower than anticipated rates $[15,16]$. Comparing local radiotherapy with and without regional radiotherapy in the non-NAC, pN1, post-operative setting, the European Organisation for Research and Treatment of Cancer trial 22922/10925 and the National Cancer Institute of Cancer MA-20 trial reported 12 and $8.4 \%$ lymphedema rates with regional radiotherapy, versus 10.5 and $4.5 \%$ without regional radiotherapy at median follow up of 10.9 and 9.5 years respectively $[15,16]$.

This study is a retrospective analysis of prospectively collected data and as such has weaknesses. There was much heterogeneity of chemotherapy, HER2 amplification testing and targeted therapy over the data collection period and these areas have evolved considerably. Poorly represented subgroups in this cohort are $\mathrm{cN}$ stage $>1$, ypN stage $>1$, combined yp stage $>$ II, inflammatory breast cancer and those with non-ductal histology.

\section{Conclusion}

Local radiotherapy following NAC and oncological resection for clinical stage II and III breast cancer may be a reasonable option in selected patients considered at low risk of harbouring regional disease. Such a hypothesis however requires confirmation from high quality, randomised control trials and recruitment into studies, such as NSABP B-51 for those converting to ypN0, is encouraged. Whilst distant recurrence is the dominant relapse pattern, regional recurrence as a component of first failure was not uncommon.

\section{Abbreviations}

AJCC, American Joint Committee of Cancer; CT, computed tomography; DFS, disease free survival; DM, distant metastasis; ER, estrogen receptor; FFDM, freedom from distant metastasis; FFLRR, freedom from locoregional recurrence; HER2, human epidermal receptor 2; LR, local recurrence; LRR, locoregional recurrence; NAC, neoadjuvant chemotherapy; OS, overall survival; $\mathrm{pCR}$, pathological complete response; RR, regional recurrence; SCF, supraclavicular fossa

\section{Acknowledgements}

The Department of Breast Cancer Research is acknowledged for non-financial assistance.

\section{Funding}

There was no additional funding beyond that of the department which contributed to the collection of data and production of the manuscript. The Breast Oncology Department is funded through the National Health Service, United Kingdom.

\section{Availability of data and materials}

De-identified datasets can be retrieved from the principle author upon formal request, but are unable to be stored on a public repository.

\section{Authors' contributions}

RW - writing of manuscript; TD - medical statistics; AM - formulation of research question, supervision and revision of manuscript. All authors read and approved the final manuscript.

\section{Competing interests}

The authors declare that they have no competing interests.

\section{Consent for publication}

Not applicable.

\section{Ethics approval and consent to participate}

Advice for this project was sought from the Mount Vernon Cancer Centre Research and Development Unit. As this research was limited to the secondary use of information previously collected in the course of normal care (without an intention to use it for research at the time of collection) it was excluded from requiring ethics approval.

\section{Author details}

${ }^{1}$ University of Western Australia, Nedlands, Australia. ${ }^{2}$ Breast Cancer Research Unit, Mt Vernon Cancer Centre, Middlesex, UK.

Received: 28 February 2016 Accepted: 15 July 2016

Published online: 26 July 2016

\section{References}

1. Buchholz TA, Lehman CD, Harris JR, et al. Statement of the science concerning locoregional treatments after preoperative chemotherapy for breast cancer: a National Cancer Institute conference. J Clin Oncol. 2008:26:791-7.

2. Bellon JR, Wong JS, Burstein HJ. Should response to preoperative chemotherapy affect radiotherapy recommendations after mastectomy for stage II breast cancer? J Clin Oncol. 2012;30:3916-20

3. Daveau C, Stevens D, Brain E, et al. Is regional lymph node irradiation necessary in stage II to III breast cancer patients with negative pathologic node status after neoadjuvant chemotherapy? Int J Radiat Oncol, Biol, Phys. 2010;78:337-42.

4. Min SY, Lee SJ, Shin KH, et al. Locoregional recurrence of breast cancer in patients treated with breast conservation surgery and radiotherapy following neoadjuvant chemotherapy. Int J Radiat Oncol, Biol, Phys. 2011:81:e697-705.

5. Shim S, Won P, Seong Jae H, et al. The Role of Postmastectomy Radiation Therapy (PMRT) in Clinical Stage II-III Breast Cancer Patients With pNo After Neoadjuvant Chemotherapy: A Multicenter, Retrospective Study (KROG 12-05). Int J Radiat Oncol. 2013:87:S221.

6. Bae SH, Park W, Huh SJ, et al. Radiation treatment in pathologic n0-n1 patients treated with neoadjuvant chemotherapy followed by surgery for locally advanced breast cancer. J Breast Cancer. 2012;15:329-36.

7. Haffty BG, McCall LM, Ballman KV, et al. Patterns of local-regional management following neoadjuvant chemotherapy in breast cancer: Results from ACOSOG Z1071 (Alliance). Int J Radiat Oncol. 2015;94:493-502.

8. Edge S, Byrd D, Compton C, et al. AJCC cancer staging manual. 2010.

9. Bentzen SM, Agrawal RK, Aird EGA, et al. The UK Standardisation of Breast Radiotherapy (START) Trial B of radiotherapy hypofractionation for treatment of early breast cancer: a randomised trial. Lancet. 2008;371:1098-107.

10. White J, Mamounas E. Locoregional radiotherapy in patients with breast cancer responding to neoadjuvant chemotherapy: a paradigm for treatment individualization. J Clin Oncol. 2014:32:494-5.

11. Mamounas EP, Anderson SJ, Dignam JJ, et al. Predictors of locoregional recurrence after neoadjuvant chemotherapy: results from combined analysis of National Surgical Adjuvant Breast and Bowel Project B-18 and B-27. J Clin Oncol. 2012;30:3960-6.

12. Huang EH, Tucker SL, Strom EA, et al. Predictors of locoregional recurrence in patients with locally advanced breast cancer treated with neoadjuvant chemotherapy, mastectomy, and radiotherapy. Int I Radiat Oncol, Biol, Phys. 2005;62:351-7.

13. Nielsen HM, Overgaard M, Grau C, et al. Loco-regional recurrence after mastectomy in high-risk breast cancer-risk and prognosis. An analysis of patients from the DBCG 82 b\&c randomization trials. Radiother Oncol. 2006; 79:147-55. 
14. Le Scodan R, Selz J, Stevens D, et al. Radiotherapy for stage II and stage III breast cancer patients with negative lymph nodes after preoperative chemotherapy and mastectomy. Int J Radiat Oncol, Biol, Phys. 2012;82:e1-7.

15. Poortmans PM, Collette S, Kirkove C, et al. Internal Mammary and Medial Supraclavicular Irradiation in Breast Cancer. N Engl J Med. 2015;373:317-27.

16. Whelan TJ, Olivotto IA, Parulekar WR, et al. Regional Nodal Irradiation in Early-Stage Breast Cancer. N Engl J Med. 2015;373:307-16.

Submit your next manuscript to BioMed Central and we will help you at every step:

- We accept pre-submission inquiries

- Our selector tool helps you to find the most relevant journal

- We provide round the clock customer support

- Convenient online submission

- Thorough peer review

- Inclusion in PubMed and all major indexing services

- Maximum visibility for your research

Submit your manuscript at www.biomedcentral.com/submit
Biomed Central 